ZIBELINE INTERNATIONAL

ISSN: 2616-4302 (Online)

CODEN : AMMCFL

\title{
DESIGN AND EXPERIMENT OF RECIPROCATING DOUBLE TRACK STRAIGHT LINE CONVEYOR
}

\author{
Xiaochao Tian ${ }^{1}$, Qinghua $\mathrm{Li}^{1}$, Chunshan $\mathrm{He}^{1}$, Yunguang Cai ${ }^{1}$, Yan Zhang ${ }^{1}$, Zhigang Yang ${ }^{2}$ \\ ${ }^{1}$ School of Mechanical and vehicle Engineering, Changchun University, Changchun 130022, China \\ ${ }^{2}$ School of Mechanical Science and Engineering, Jilin University, Changchun 130025, China \\ *Corresponding Author Email: tianxch@ccu.edu.cn
}

This is an open access article distributed under the Creative Commons Attribution License, which permits unrestricted use, distribution, and reproduction in any medium, provided the original work is properly cited

\begin{tabular}{|c|c|}
\hline ARTICLE DETAILS & ABSTRACT \\
\hline $\begin{array}{l}\text { Article History: } \\
\text { Received } 12 \text { November } 2017 \\
\text { Accepted } 12 \text { December } 2017 \\
\text { Available online 1 January } 2018\end{array}$ & $\begin{array}{l}\text { In order to realize the bidirectional transportation of materials, a reciprocating double track linear conveyor is } \\
\text { designed in this paper. The conveyer uses the piezoelectric vibrator as its driving force source to drive and uses the } \\
\text { linear structure to realize the spiral conveying function. Conveyors have the capability to convey materials with a small } \\
\text { volume. Firstly, the structure of the conveyor is introduced. Then, the modal analysis and simulation of the piezoelectric } \\
\text { vibrator and the conveying system are carried out. We find out that the system is worked under the two-order mode } \\
\text { and obtained the working principle of the system. Finally, make prototype and test it, the system voltage and frequency } \\
\text { characteristics were obtained. At the same time, the consistency of the dual rail conveying materials under resonance } \\
\text { condition is tested. The results show that the system performance and delivery consistency are better. The conveyer } \\
\text { has wide application prospect in narrow space material transportation. }\end{array}$ \\
\hline
\end{tabular}

\section{KEYWORDS}

Reciprocating conveyor, piezoelectric drive, vibration mode, resonance frequency

\section{INTRODUCTION}

Conveyor is an automatic feeding device for sorting, sorting and directional sorting. It has the advantages of simple structure, convenient application, high work efficiency, etc. Conveyor has been used more and more widely in the automatic production system such as electronic component, medical apparatus and light industry [1-3]. But with the development of industrialization, the demand for material conveyor, is no longer the initial realization entire column feeding function is so simple, but in more and more fields and the environment need to lower power consumption, less noise, conveying more sophisticated and more beautiful appearance, and meet the requirements of material delivery in a narrow space in.

In the practical application of piezoelectric conveyor [4-6], general spiral feeder and the feeder line with the use of two jointly form feeding automation equipment. In this system, the spiral feeder is mainly played the role of storage and array, convey materials to the feeder line. Linear feeder line is the realization of long distance transmission function, does not have the material screening and the whole function. Two kinds of feeder used in conjunction with each other will realize the whole automation equipment material column and transportation, but the delivery system is limited in the use of space.

This paper designs a kind of dual channel reciprocating linear piezoelectric material conveyor, material for reciprocating motion in two straight tracks. At the same time in the long-distance transportation of screening and the whole function, can be a substitute for a conveyor device of the conveying system, greatly saves cost and saving the automation equipment room.

\section{STRUCTURAL DESIGN AND MODAL ANALYSIS}

\subsection{Structural design}

The design of the dual channel piezoelectric linear reciprocating conveyor 3D model as shown in Figure 1, is mainly composed of a top plate, a supporting spring plate, piezoelectric vibrator, the adjusting block and the base made of. For the base fixed on the table, the top plate is fixed above the straight track, for the storage and the whole material conveying, adjusting block is used to adjust the overall position. The structure can realize the function of material screening and transportation with smaller volume and can work in a narrow space.

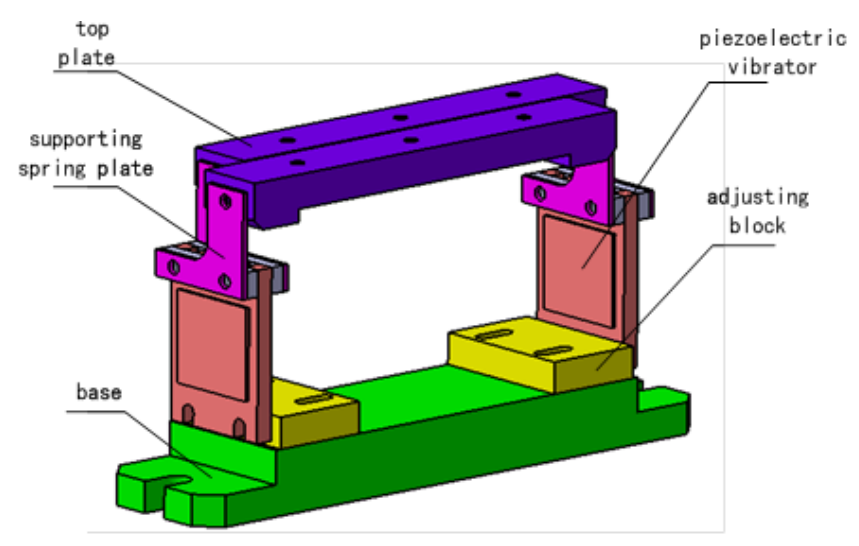

Figure 1: 3D model of reciprocating linear conveyor

\subsection{Modal simulation of piezoelectric vibrators}

The simulation software ABAQUS is used to simulate the piezoelectric vibrator. In this paper, the bimorph piezoelectric vibrator is used as the 
driving force, and its vibration characteristics directly affect the performance of the system. In order to make a more intuitive analysis of the dynamic characteristics of the piezoelectric vibrator and to predict the vibration shape of the system at first, the vibration modal analysis of the piezoelectric vibrator is carried out by using the finite element analysis software ABAQUS. The parameters of piezoelectric vibrators are as follows:

The parameters of piezoelectric ceramics: density $\rho_{p}=7.5 \times 10^{3} \mathrm{~kg} / \mathrm{m}^{3}$, dielectric constant matrix $\left(\varepsilon \times 10^{-9} \mathrm{~F} / \mathrm{m}\right)$, piezoelectric constant matrix $\left(e \times C / m^{2}\right)$, piezoelectric elastic coefficient matrix $\left(c \times 10^{10} \mathrm{~N} / \mathrm{m}^{2}\right)$ respectively as:

$[\varepsilon]=\left[\begin{array}{ccc}7.124 & 0 & 0 \\ 0 & 7.124 & 0 \\ 0 & 0 & 5.841\end{array}\right][e]=\left[\begin{array}{ccc}0 & 0 & -4.1 \\ 0 & 0 & -4.1 \\ 0 & 0 & 14.1 \\ 0 & 0 & 0 \\ 0 & 10.5 & 0 \\ 10.5 & 0 & 0\end{array}\right][c]=\left[\begin{array}{cccccc}13.2 & 7.1 & 7.3 & 0 & 0 & 0 \\ 7.1 & 13.2 & 7.3 & 0 & 0 & 0 \\ 7.3 & 7.3 & 11.5 & 0 & 0 & 0 \\ 0 & 0 & 0 & 3 & 0 & 0 \\ 0 & 0 & 0 & 0 & 2.6 & 0 \\ 0 & 0 & 0 & 0 & 0 & 2.6\end{array}\right]$

The material parameters of the metal substrate $(65 \mathrm{Mn})$ of piezoelectric vibrators are: density $\rho_{65 \mathrm{Mn}}=7.85 \times 10^{3} \mathrm{~kg} / \mathrm{m}^{3}$, modulus of elasticity $E_{65 M n}=2.06 \times 10^{11} \mathrm{~Pa}$, Poisson ratio $u_{65 M n}=0.3$.

Through simulation modal analysis, the four vibration modes of the piezoelectric vibrator are obtained, as shown in figure 2. From the vibration mode nephogram, it can be seen that the first order vibration mode is the flexural vibration along the length direction; the second order vibration mode is torsional vibration; the third order vibration mode and the fourth order vibration mode are messy and meaningless. From the system working form, the piezoelectric vibrator operates in the two order mode.

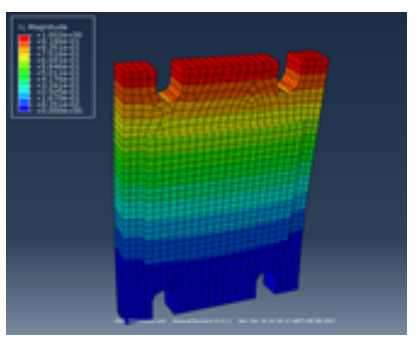

a) First order vibration modes vibration mode

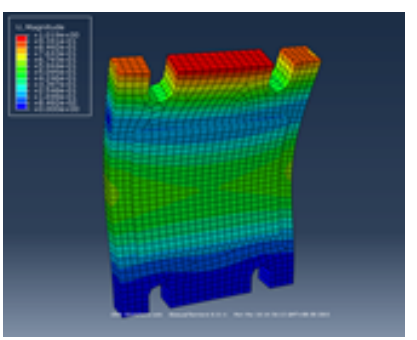

c) Third order vibration modes

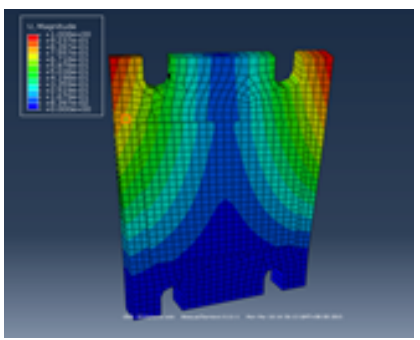

b) Second order vibration mode

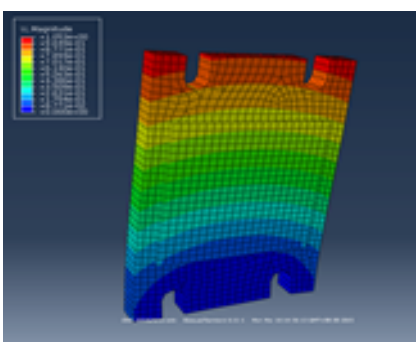

d) Fourth order vibration mode
Figure 2: Modal of piezoelectric vibrator

\subsection{Whole machine modal analysis}

The system is tested by simulation software. The grid is divided, the boundary conditions are set up, and the material parameters of each part of the system are set up. The first five order vibration modes and the modal frequencies of the conveying system are obtained, as shown in Figure 3. Materials and parameters of each part of the vibration conveying system are shown in Table 1, which mainly include 65Mn spring steel, 45 steel and hard aluminum alloys.

Table 1: Materials and Parameters of Each Part of the Conveying System

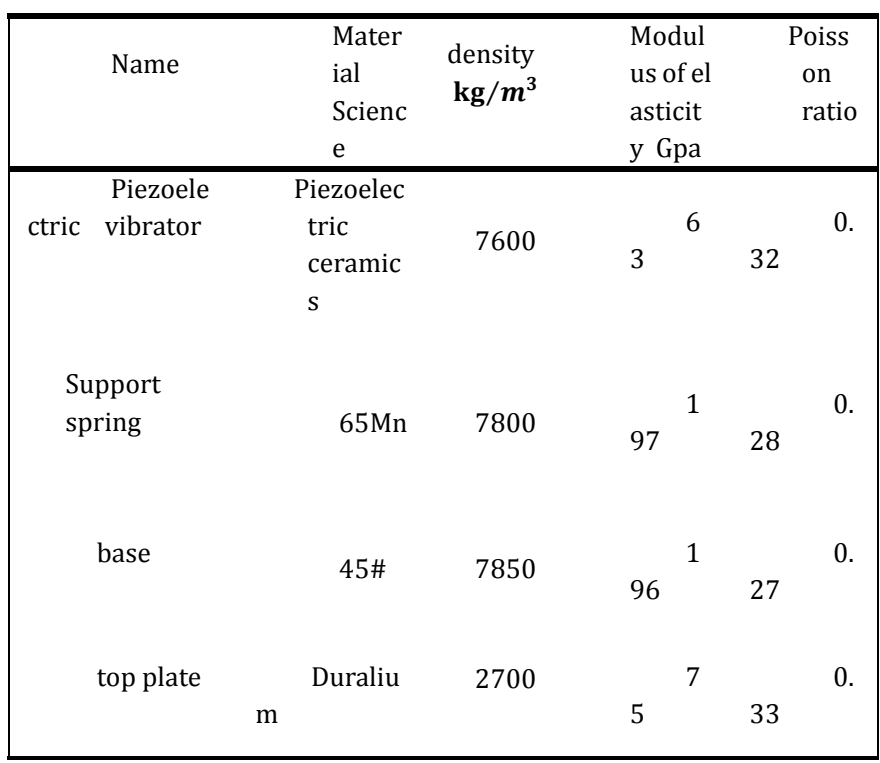

From the modal images can be seen in the first order vibration shape for pure bending vibration, second order vibration shape and torsional vibration, natural frequency is $151.4 \mathrm{~Hz}$, third stage, fourth stage and fifth modes rather messy. The second order vibration mode agrees well with the simulation analysis of the vibration modes of the piezoelectric vibrator, so the conveyor operates normally in the second order torsional mode.

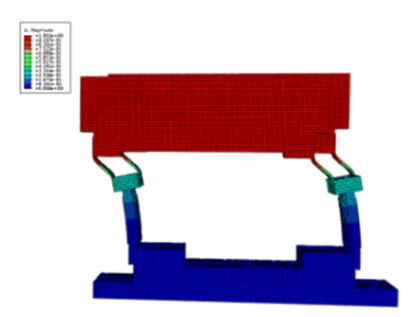

\section{t}

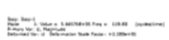

a) First order vibration model

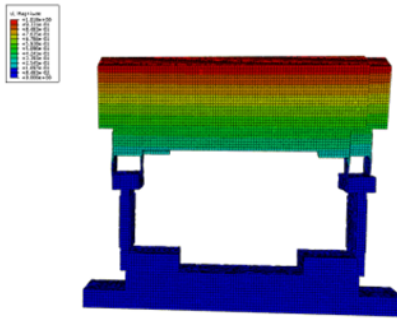

\section{if $=-\cdots$}

c) Third order vibration model

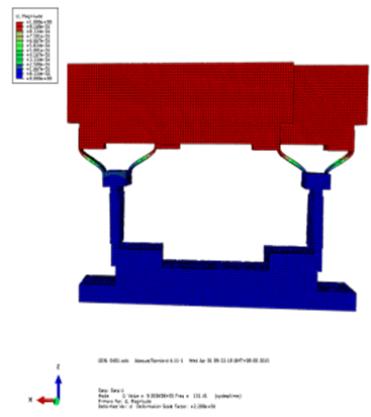

b) Second order vibration model

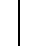
(1) . 


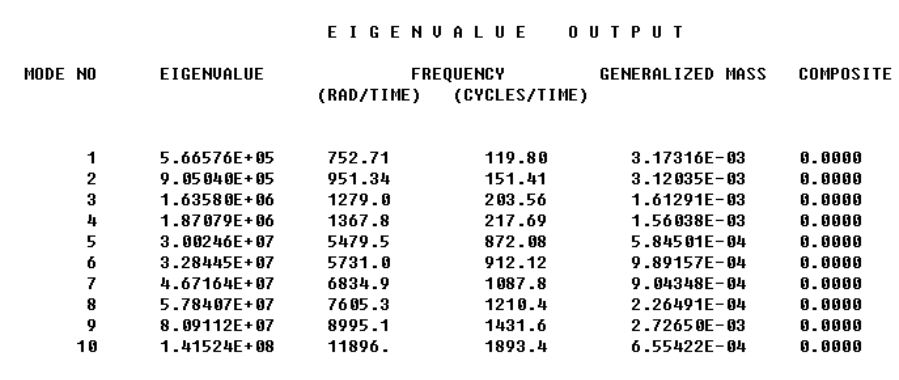

Figure 4: Modal frequency of system order

\subsubsection{Working principle}

From the analysis of vibration modal simulation of piezoelectric vibrator and conveyor system, we know that, the bimorph piezoelectric vibrator under the action of alternating signals, can torsional alternating deformation occur, and make the supporting spring produce elastic deformation, to drive the top plate and double track vibration level approximation. As the linear conveyor in the two-vibration mode of piezoelectric bimorph, one end of which is connected with the supporting spring do exist torsional vibration, friction between the material and the material disc, the piezoelectric vibrator is less than the return reverse forward acceleration, so the material and material by friction between the discs to move forward, because the top two vibration phase instead, so as to realize the two top conveying cycle. The special design of the straight track enables the material to be screened and arranged throughout its cycle, and the final material is delivered to the specified docking device in a specified manner.

\section{EXPERIMENTAL METHOD}

This experiment uses M3 metal small nut as the system test object, the method is to measure the material in the straight track double track entire movement time which consumed. In order to ensure the accuracy of the experimental results, the average value method is adopted, that is, each experiment is repeated five times without changing the experimental environment and parameters, and the algebraic average of the five measurements is obtained. In order to express the speed of feeding more visually, the measured data is converted to the number of materials transported in unit time.

The actual determination method of conveying speed: the conveyor is fixed on the bench and is connected with the piezoelectric controller. After the power is switched on, adjust to proper voltage and frequency, and cause the conveyor to resonate. Measuring the time, it takes for the material to move from one end of the straight track to the other. Finally, the relationship between the feeding speed and the voltage and frequency is plotted. The test device is shown in figure 5 .

The actual measuring method of system amplitude: using laser micrometer to measure amplitude, firstly, the conveyer is fixed on the experimental platform, connected with the piezoelectric controller, and connected with the power supply. Through the piezoelectric controller to change the driving frequency and voltage, point the laser head to the measuring point, measure the amplitude of the selected frequency value or voltage value, record the data, draw the relationship curve, and test the device as shown in figure 6 .
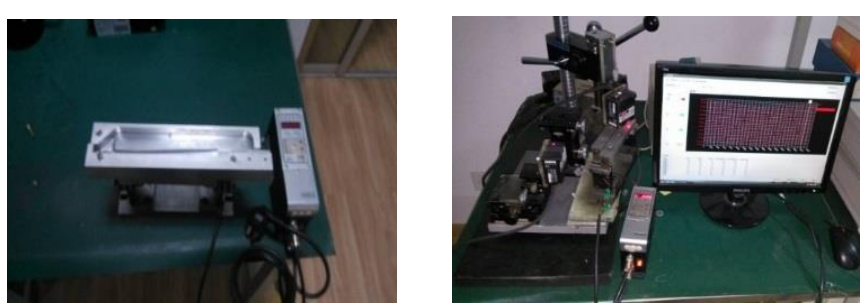

Figure 5: Speed test device diagram test

\subsection{Conveyor frequency characteristic}

Drive the voltage to $200 \mathrm{~V}$, change the drive frequency of the system, in the second phase of the conveyor frequency near the natural frequency to measure the speed of transmission, the measurement results shown in figure 7 . At the same time, the amplitude changes of the conveyer under this process are measured by laser micrometer, and the measurement result is shown in figure 8 .

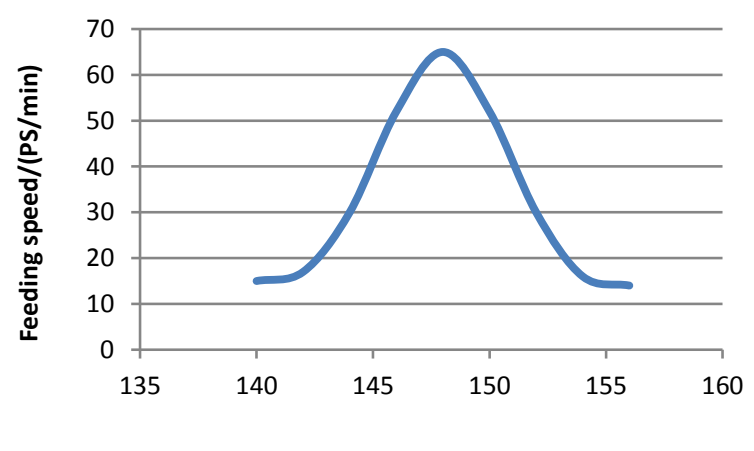

Driving frequency/Hz

Figure 7: Relationship between drive frequency and conveying speed

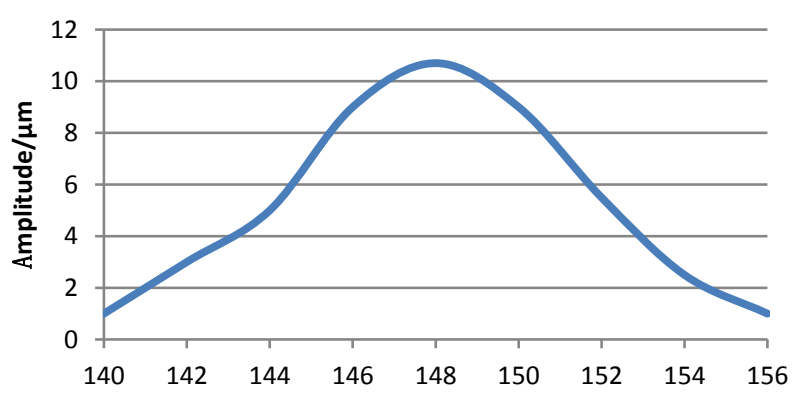

Driving frequency/Hz

Figure 8: Relationship between drive frequency and system amplitude

As can be seen from Figure 7, the conveying speed of the conveyor is affected by the driving frequency. When the driving frequency is near $148 \mathrm{~Hz}$, the speed of the conveyor two tracks is maximum, reaching 65 $/ \mathrm{min}$, at which time the system reaches a resonance state. When the driving frequency deviates from the resonant frequency, the conveying speed decreases obviously.

As can be seen from figure 8, the trend of the amplitude curve is similar to that of the transport velocity curve. When the driving frequency reaches $148 \mathrm{~Hz}$, the amplitude reaches the maximum value of $11 \mathrm{mu} \mathrm{m}$. When the driving frequency deviates from the resonant frequency, the amplitude of the conveyor is obviously decreased, and the frequency domain and the conveying speed are almost the same.

\subsection{Conveyor voltage characteristic}

First, the voltage of the piezoelectric controller is tuned to the resonant frequency $148 \mathrm{~Hz}$, the voltage is zero, and the voltage increases at $20 \mathrm{~V}$ each to determine the corresponding speed of delivery, that is, the time from the end of the rail to the other end of the material. Then, the track vibration amplitudes of different voltages are tested in turn by means of a laser micrometer, and the curves of voltage versus speed and amplitude are obtained, as shown in Figure 9 and figure 10, respectively. 


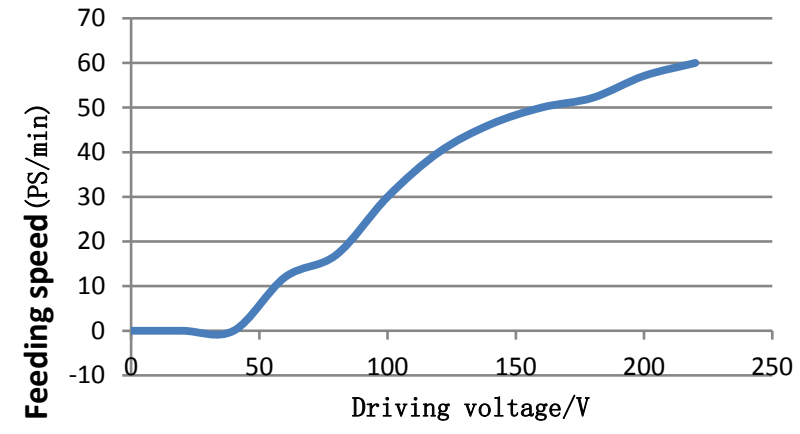

Figure 9: Relationship between transmission speed and driving voltage

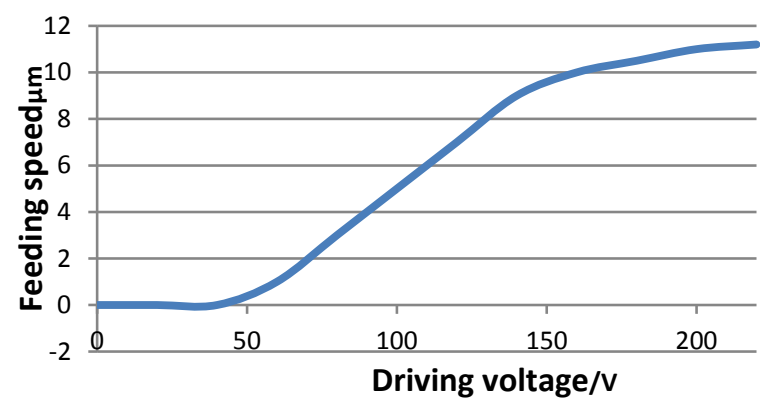

Figure 10: Relationship between amplitude and driving voltage

As can be seen from Fig. 9, when the voltage is extremely low, the conveyor feed rate is almost 0 , and cannot be fed. When the voltage increases, the feed capacity of the conveyor increases gradually. With the increase of the voltage, the feed rate also increases, and its relationship is approximately linear increase. When the voltage increases to $200 \mathrm{~V}$, the transport speed is $54 / \mathrm{min}$.

As can be seen from Fig. 10, when the voltage of the piezoelectric controller is very low, the conveyor is almost free of vibration, and the data measured by the laser micrometer is very small and can be neglected. When the voltage reaches a certain value, the vibration amplitude of the conveyor begins to increase, but the amplitude is still small and can hardly be fed at this time. When the voltage continues to increase, the amplitude continues to increase, and the feeding capacity increases gradually, and basically linearly. When the voltage is increased to $200 \mathrm{~V}$, the amplitude is $13 \mu \mathrm{m}$.

\subsection{Consistency of conveying speed}

In order to ensure the material transportation, the conveyor also needs to investigate the consistency of the material speed of the two-straight track. That is the natural frequency of the driving frequency conveyor reached two modes, the two-track speed difference must be maintained in a certain range, otherwise the material cannot achieve effective circulation in a straight line, prone to accumulation of clogging, conveying performance conveyor.

The first driving voltage to $200 \mathrm{~V}$ conveyor, while driving near the frequency to two order natural frequency, frequency characteristics in the frequency domain are effective, feeding and discharging test track rail transportation speed, the test results are as follows:
Table 2: Data Sheet for Track Speed Consistency of Incoming and Outgoing Tracks

\begin{tabular}{|c|c|c|c|c|c|c|c|c|c|}
\hline $\begin{array}{c}\text { frequency (Hz) } \\
\text { speed (ps/min }\end{array}$ & 141 & 143 & 145 & 147 & 149 & 151 & 153 & 155 & 157 \\
\hline Feed track & 16 & 18 & 31 & 53 & 66 & 49 & 24 & 9 & 6 \\
\hline Discharge track & 7 & 10 & 25 & 50 & 65 & 54 & 32 & 17 & 15 \\
\hline Velocity difference & 9 & 8 & 6 & 5 & 4 & 5 & 8 & 8 & 9 \\
\hline
\end{tabular}

As can be seen from the above table, when the driving frequency reaches the natural frequency near the two orders, the speed uniformity of the conveyor is the best. When the driving frequency deviates from the twoorder natural frequency, its consistency becomes worse and worse, and the speed of the feeding track decreases gradually, and the speed of the discharge track gradually increases.

\section{CONCLUSION}

A reciprocating double track linear piezoelectric conveyor is designed in this paper. The system of the rectangular piezoelectric vibrator and the whole machine works in the second stage mode, and the natural frequency of the second stage is $151.4 \mathrm{~Hz}$. It has a certain error with the resonance frequency $148 \mathrm{~Hz}$ of the experimental test, but basically agrees with it. The experimental tests show that the conveying speed and amplitude increase with the increase of voltage. With the increase of the driving frequency, the system increases first and then decreases, and the system works better under resonance. At the same time, the consistency of the two linear orbits is tested. The results show that the two linear track agrees well in the resonance state. When the deviation from the resonance interval, the consistency is poor.

\section{ACKNOWLEDGMENTS}

This work is funded by the National Science Foundation of China (51705031) and Scientific research project of the Education Department of Jilin Provincial(JJKH20170489KJ).

\section{REFERENCE}

[1] Xiliang, Z., Xin, L. 2003. Characteristics of an Electromagnetic Vibration Feeder and Its Application [J]. Transaction of the Chinese Society for Agricultural Machinery, 34 (5), 92-95.

[2] Yunchi, W. 2003. Present situation and development of vibration feeding equipment in China [J]. Coal Quality Technology, 4, 29-31.

[3] Jiang, S. 2012. Current advances and development trends of vibration feeder [J]. Refitting and Maintenance, 7, 178-180.

[4] Mal'tsev V.A., Yudin A.V. 2003. Influence of parameters of the vibrating feeder - screen on speed of the vibrating transportation of rock under nonsteady conditions of the operating toolmovement [J]. Izvestiya Vysshikh Uchebnykh Zavedenii, 5, 71-76.

[5] Aoyagi, M., Beeby, S.P., White, N.M. 2002. A novel multi-degree-offreedom thick-film ultrasonic motor [J]. Ultrasonic, Ferroelectrics and Frequency Control, IEEE Transactions on, 49 (2), 151-158.

[7] Yan-hu, S., Jiang, S., Zhigang, Y. 2014. Design and experiment of piezoelectric-drive vibratory Feeder [J]. Optics and Precision Engineering, 22 (7), 1828-1833. 